\title{
DIVISIONS OF SPACE BY PARALLELS
}

BY

\author{
G. L. ALEXANDERSON AND JOHN E. WETZEL
}

\begin{abstract}
An arrangement of hyperplanes in $\mathbb{E}^{d}$ is a "plaid" provided its hyperplanes form no multiple flats of intersection and lie in parallel families that are in general position. We develop some geometrically natural formulas for the number of $r$-faces that are formed by such an arrangement.
\end{abstract}

1. Introduction. An arrangement of $n$ hyperplanes in $\mathbb{E}^{d}$ that lie in $s \geqslant d$ parallel families is a "plaid" provided it forms no multiple flats of intersection and its $s$ parallel families are in general position. The notion is due to J. Steiner, who in 1826, in his third published paper [8], found elegant formulas involving the elementary symmetric functions for the number of cells, bounded or unrestricted, that are formed by plaids in $\mathbb{E}^{2}$ and $\mathbb{E}^{3}$. T. Zaslavsky [9] has given analogous general formulas for the number of faces of each dimension that are formed by a plaid in $\mathbb{E}^{d}$. The Steiner-Zaslavsky formulas and other preliminaries are presented in $\$ 2$.

A plaid with prescribed parallel structure can be generated by displacing $n-s$ hyperplanes in a generic arrangement of $n$ hyperplanes or by inserting $n-s$ parallels into a generic arrangement of $s$ hyperplanes so as to create the desired parallel families. Each point of view suggests formulas for the number of $r$-faces that are formed by the plaid.

The former leads to formulas that enumerate the $r$-faces in terms of the loss from general position. These "decrement" formulas, which we develop in $\$ 3$, are readily deduced from the Steiner-Zaslavsky formulas with the help of the general addition formula for the binomial coefficients.

The latter point of view leads to formulas that enumerate the $r$-faces in terms of the gain from general position. In §6, after two sections of geometric preliminaries, we present a direct, geometric derivation of these "increment" formulas that spells out the geometric meaning of each term. An ex post facto algebraic reduction of these formulas to the Steiner-Zaslavsky formulas is also given, in $§ 7$.

$\S 8$ contains an algebraic proof of a different, unpublished face-count formula due to Zaslavsky that is somewhat similar to the increment formula but has quite different geometric roots.

Received by the editors August 9, 1984 and, in revised form, January 4, 1985. Some of these results were presented by the first author at the special session on Combinatorial Identities at the AMS Summer Meeting in Toronto in August 1976, and some were presented by the second author at an AMS Regional Meeting at Purdue University in October 1977.

1980 Mathematics Subject Classification. Primary 51M20; Secondary 05A19.

Key words and phrases. Arrangements of hyperplanes, division of space, parallel hyperplanes, plaid, symmetric functions. 
Finally, we want to express our gratitude to Thomas Zaslavsky, whose insightful suggestions contributed significantly to the paper.

2. Plaids in $\mathbb{E}^{d}$. We begin by recalling some definitions and results about arrangements in Euclidean $d$-space $\mathbb{E}^{d}$, with $d \geqslant 1$. As general references for this material, see Grünbaum [5], McMullen and Shephard [6], and Zaslavsky [10].

A Euclidean $d$-arrangement $\mathscr{E}$, i.e., a finite set of hyperplanes in $\mathbb{E}^{d}$, is nondegenerate if some $d$ of its hyperplanes have a point as their intersection. This condition means that $\mathscr{E}$ is truly $d$-dimensional in the sense that it does not have constant cross-section. Unless the contrary is explicitly indicated, we always assume that the arrangements under consideration are nondegenerate, so that in particular, $n=|\mathscr{E}|$ $\geqslant d$.

An arrangement $\mathscr{E}$ is (Euclidean-) simple if for each $k$ with $0 \leqslant k \leqslant d-2$, no more than the obligatory $d-k$ hyperplanes of $\mathscr{E}$ pass through any $k$-flat. A simple arrangement has no multiple flats, but flats of various dimensions may be parallel (i.e., disjoint). If there are no such parallelisms, that is, if $\bigcap(\mathscr{H}) \neq \varnothing$ for every collection $\mathscr{H} \subseteq \mathscr{E}$ with $2 \leqslant|\mathscr{H}| \leqslant d$, then the simple arrangement $\mathscr{E}$ is said to be generic, or in general position. In what follows, we denote a generic arrangement of $n$ hyperplanes in $\mathbb{E}^{d}$ by $\mathscr{G}^{d}(n)$.

The $n=|\mathscr{E}|$ hyperplanes of the $d$-arrangement $\mathscr{E}$ fall into $s \geqslant d$ parallel families $\pi_{1}$, $\pi_{2}, \ldots, \pi_{s}$ having $n_{1}, n_{2}, \ldots, n_{s}$ hyperplanes, respectively; we permit $n_{i}=1$ to accommodate hyperplanes having no parallel partners. We call the $s$-vector $\mathbf{n}=$ $\left\langle n_{1}, n_{2}, \ldots, n_{s}\right\rangle$ the Steiner data of $\mathscr{E}$. A frame of $\mathscr{E}$ is a set of $s$ hyperplanes of $\mathscr{E}$, one from each parallel family. A plaid in $\mathbb{E}^{d}$ is a simple $d$-arrangement that has a generic frame.

Formulas for the number $f_{r}\left(\mathscr{P}^{d}(\mathbf{n})\right)$ of $r$-faces formed by a plaid $\mathscr{P}^{d}(\mathbf{n})$ with Steiner data $\mathbf{n}$ were given by Steiner [8] (for $r=d=2,3$ ), Zaslavsky [9], and Alexanderson and Wetzel [2], as follows. For each $j$ with $0 \leqslant j \leqslant s$, let $\sigma_{j}=\sigma_{j}(\mathbf{n})$ be the $j$ th elementary symmetric function on the variables $n_{1}, n_{2}, \ldots, n_{s}$.

THEOREM 1. For each $r$ with $0 \leqslant r \leqslant d$, a plaid $\mathscr{P}^{d}(\mathbf{n})$ with Steiner data $\mathbf{n}$ partitions $\mathbb{E}^{d}$ into

$$
f_{r}\left(\mathscr{P}^{d}(\mathbf{n})\right)=\sum_{j=d-r}^{d}\left(\begin{array}{c}
j \\
d-r
\end{array}\right) \sigma_{j}(\mathbf{n})
$$

r-faces, of which

$$
f_{r}^{\prime}\left(\mathscr{P}^{d}(\mathbf{n})\right)=\sum_{j=d-r}^{d}(-1)^{d-j}\left(\begin{array}{c}
j \\
d-r
\end{array}\right) \boldsymbol{\sigma}_{j}(\mathbf{n})
$$

are bounded.

As in (1) and (2), it frequently happens that a formula for $f_{r}^{\prime}(\mathscr{E})$ can be obtained from that for $f_{r}(\mathscr{E})$ by simply alternating the signs. (For an example to the contrary, see Theorem 8 of Alexanderson and Wetzel [1].) We take advantage of this symmetry and combine (1) and (2) into a single formula (3) by introducing neutral notation and language as follows. We write $\varphi_{r}$ for $f_{r}^{\prime}$ or $f_{r}$ and take $\eta$ equal to -1 or +1 in the bounded or unrestricted cases, respectively, and in the former case we 
construe " $r$-face" to mean "bounded $r$-face." In this language, the formulas of Theorem 1 say that a plaid $\mathscr{P}^{d}(\mathbf{n})$ forms

$$
\varphi_{r}\left(\mathscr{P}^{d}(\mathbf{n})\right)=\sum_{j=d-r}^{d} \eta^{d-j}\left(\begin{array}{c}
j \\
d-r
\end{array}\right) \sigma_{j}(\mathbf{n})
$$

$r$-faces. We shall employ this language, where appropriate, throughout.

Formula (3) specializes to formulas given by Buck [3], Zaslavsky [10], and many others for generic $d$-arrangements.

COROllaRY 2. For each $r$ with $0 \leqslant r \leqslant d$, a generic arrangement $\mathscr{G}^{d}(n)$ of $n$ hyperplanes partitions $\mathbb{E}^{d}$ into

$$
\varphi_{r}\left(\mathscr{G}^{d}(n)\right)=\sum_{j=d-r}^{d} \eta^{d-j}\left(\begin{array}{c}
j \\
d-r
\end{array}\right)\left(\begin{array}{l}
n \\
j
\end{array}\right)\left(\begin{array}{c}
n \\
d-r
\end{array}\right) \sum_{k=0}^{r} \eta^{r-k}\left(\begin{array}{c}
n-d+r \\
k
\end{array}\right)
$$

r-faces.

The second sum arises from the first by refactoring the trinomial coefficient $\left(\begin{array}{c}n \\ n-j, d-r\end{array}\right)$. It is worth noting that in the bounded case the second sum has a simple closed form (see Gould [4, formula 1.5]):

$$
f_{r}^{\prime}\left(\mathscr{G}^{d}(n)\right)=\left(\begin{array}{c}
n \\
d-r
\end{array}\right)\left(\begin{array}{c}
n-d+r-1 \\
r
\end{array}\right) .
$$

It will be convenient to abbreviate $\varphi_{r}\left(\mathscr{G}^{d}(n)\right)$ to $G_{r}^{d}(n)$ and to take $G_{r}^{d}(n)=0$ when the condition $0 \leqslant r \leqslant d$ fails to hold. Note that formula (4) is also correct for $d=0$ provided we take $G_{0}^{0}(n)=1$.

For the purpose of illustration we consider one special case, which we carry throughout. We call a plaid $\mathscr{P}^{d}(\mathbf{n})$ skew provided $n_{1}=m \geqslant 1$ and $n_{i}=1$ for $2 \leqslant i \leqslant s$. We denote such a plaid by $\mathscr{S}^{d}(m)$. Evidently $n=s+m-1$ and

$$
\boldsymbol{\sigma}_{j}(\mathbf{n})=(m-1)\left(\begin{array}{l}
s-1 \\
j-1
\end{array}\right)+\left(\begin{array}{l}
s \\
j
\end{array}\right)
$$

and (3) and (4) give the increment formula

$$
\varphi_{r}\left(\mathscr{S}^{d}(m)\right)=G_{r}^{d}(s)+(m-1) \sum_{j=d-r}^{d} \eta^{d-j}\left(\begin{array}{c}
j \\
d-r
\end{array}\right)\left(\begin{array}{l}
s-1 \\
j-1
\end{array}\right) .
$$

In the bounded case the sum can be given in closed form (as in the argument for (5)), and we find the formula

$$
\begin{aligned}
f_{r}^{\prime}\left(\mathscr{S}^{d}(m)\right) & =f_{r}^{\prime}\left(\mathscr{G}^{d}(s)\right) \\
& +(m-1)\left[\left(\begin{array}{c}
s \\
d-r
\end{array}\right)\left(\begin{array}{c}
s-d+r-2 \\
r-1
\end{array}\right)+\left(\begin{array}{c}
s-1 \\
d-r-1
\end{array}\right)\left(\begin{array}{c}
s-d+r-2 \\
r
\end{array}\right)\right] .
\end{aligned}
$$

3. Decrement formulas. A plaid $\mathscr{P}^{d}(\mathbf{n})$ can be generated by deforming an arrangement $\mathscr{G}^{d}(n)$ so as to create the required parallel families. We can find formulas for the number of $r$-faces in terms of the loss from general position by combining formulas (3) and (4) with the general addition formula for the binomial coefficients. 
We begin by recalling the addition formula (cf. Alexanderson and Wetzel [1]). If $s \geqslant 1$ and if $n_{1}, n_{2}, \ldots, n_{s}$ are nonnegative integers whose sum is $n$, then

$$
\left(\begin{array}{l}
n \\
j
\end{array}\right)=\sum_{j} \prod_{i=1}^{s}\left(\begin{array}{c}
n_{i} \\
m_{i}
\end{array}\right)
$$

where the sum is over all ordered $s$-tuples $\left(m_{1}, m_{2}, \ldots, m_{s}\right)$ of nonnegative integers whose sum is $j$. Because the sum over those ordered $s$-tuples whose entries are only zeros and ones is precisely $\sigma_{j}$, formula (7) can be rewritten in the form

$$
\sigma_{j}=\left(\begin{array}{c}
n \\
j
\end{array}\right)-\sum_{j}^{*} \prod_{i=1}^{s}\left(\begin{array}{c}
n_{i} \\
m_{i}
\end{array}\right)
$$

where the sum is over all ordered $s$-tuples $\left(m_{1}, m_{2}, \ldots, m_{s}\right)$ of nonnegative integers whose sum is $j$ except those whose entries are exclusively zeros and/or ones. The formula sought is an immediate consequence of substituting (8) into (3) and using (4).

THEOREM 3 (DECREMENT FORMULA). For each $r$ with $0 \leqslant r \leqslant d$, a plaid $\mathscr{P}^{d}(\mathbf{n})$ with Steiner data $\mathbf{n}$ partitions $\mathbb{E}^{d}$ into

$$
\varphi_{r}\left(\mathscr{P}^{d}(\mathbf{n})\right)=G_{r}^{d}(n)-\sum_{j=d-r}^{d} \eta^{d-j}\left(\begin{array}{c}
j \\
d-r
\end{array}\right) \sum_{j}^{*} \prod_{i=1}^{s}\left(\begin{array}{c}
n_{i} \\
m_{i}
\end{array}\right)
$$

r-faces.

The interesting question of which $r$-faces are counted by each separate term in the decrement seems difficult.

For a skew plaid $\mathscr{S}^{d}(m)$,

$$
\sum_{j}^{*}\left(\begin{array}{c}
m \\
m_{1}
\end{array}\right)\left(\begin{array}{c}
1 \\
m_{2}
\end{array}\right) \cdots\left(\begin{array}{c}
1 \\
m_{s}
\end{array}\right)=\sum_{k=2}^{j}\left(\begin{array}{c}
m \\
k
\end{array}\right)\left(\begin{array}{c}
s-1 \\
j-k
\end{array}\right)
$$

and (9) becomes

$$
\varphi_{r}\left(\mathscr{S}^{d}(m)\right)=G_{r}^{d}(s+m-1)-\sum_{j=d-r}^{d} \eta^{d-j}\left(\begin{array}{c}
j \\
d-r
\end{array}\right) \sum_{k=2}^{j}\left(\begin{array}{l}
m \\
k
\end{array}\right)\left(\begin{array}{l}
s-1 \\
j-k
\end{array}\right) .
$$

4. Close hyperplanes. In our scrutiny of the anatomy of a plaid we shall need to know in detail what happens when a new hyperplane is inserted into an arrangement close to one of the hyperplanes of that arrangement. We develop the appropriate geometric preliminaries in this section.

Suppose that $\mathscr{E}$ is an arbitrary (nondegenerate) Euclidean $d$-arrangment of $|\mathscr{E}|=n$ $\geqslant d$ hyperplanes, and let $L^{r}$ be any $r$-flat in $\mathbb{E}^{d}, 0<r \leqslant d$. Regarding $L^{r}$ as an $\mathbb{E}^{r}$, we call

$$
\mathscr{E} \mid L^{r}=\left\{h \cap L^{r}: h \in \mathscr{E}, \operatorname{dim}\left(h \cap L^{r}\right)=r-1\right\}
$$

the $r$-arrangement induced in $L^{r}$ by $\mathscr{E}$.

LEMMA 4. The induced arrangement $\mathscr{E} \mid L^{r}$ is nondegenerate.

Proof. Let $\mathscr{H}$ be a subset of $\mathscr{E}$ so that $|\mathscr{H}|=d$ and $\cap(\mathscr{H})$ is a point that, without loss of generality, we take to be the origin. Let $L_{0}^{r}$ be a translate of $L^{r}$ that goes through the origin. It follows from the modular law that $\operatorname{dim}\left(L_{0}^{r} \cap h\right)$ is either $r$ or 
$r-1$ for each $h$ in $\mathscr{H}$. In the former case $L^{r}$ and $h$ are parallel (i.e., either $L^{r} \subseteq h$ or $\left.L^{r} \cap h=\varnothing\right)$, while in the latter case $L^{r} \cap h$ is a hyperplane of $\mathscr{E} \mid L^{r}$. There are at most $d-r$ hyperplanes of the former kind, because the $r$-flat $L_{0}^{r}$ lies in their intersection. Consequently there are at least $r$ hyperplanes of the latter kind. Let $\mathscr{K}$ be a set of exactly $r$ such hyperplanes. Then $\cap(\mathscr{K})$ is a $(d-r)$-flat that meets $L^{r}$ in a point. Hence $\mathscr{E} \mid L^{r}$ is nondegenerate.

We resume our standing assumption that arrangements are to be nondegenerate.

A hyperplane $h_{0}$ in an arrangement $\mathscr{E}$ is simple if for each $r, 0 \leqslant r \leqslant d-2$, each $r$-flat of $\mathscr{E}$ that lies in it is simple, i.e., is the intersection of exactly $d-r$ hyperplanes of $\mathscr{E}$ (including $h_{0}$ ). Note that an arrangement is simple precisely when each of its hyperplanes is simple. The crucial property possessed by a simple hyperplane is that each $r$-face of $\mathscr{E}$ that lies in it is the common face of exactly two $(r+1)$-faces of $\mathscr{E}$ that do not lie in it. It will simplify the language to take the $r$-faces to be closed, so that each $r$-face contains its lower-dimensional faces.

LEMMA 5. If $h_{0}$ is a simple hyperplane in an arrangement $\mathscr{E}$ and $F^{r}$ is an r-face, $0 \leqslant r \leqslant d-1$, in $\mathscr{E} \mid h_{0}$, then there are exactly two $(r+1)$-faces of $\mathscr{E}$ that meet $h_{0}$ in $F^{r}$, one on each side of $h_{0}$.

Proof. The $r$-flat $L^{r}=$ aff $F^{r}$ is the intersection with $h_{0}$ of exactly $d-r-1$ hyperplanes of $\mathscr{E} \backslash\left\{h_{0}\right\}$, and those hyperplanes meet in an $(r+1)$-flat $L^{r+1}$ of $\mathscr{E}$. Since $F^{r}$ is an $r$-face of $\mathscr{E} \mid L^{r+1}$, there are two $(r+1)$-faces in $\mathscr{E} \mid L^{r+1}$, one on each side of $h_{0} \cap L^{r+1}$, that meet $h_{0}$ in $F^{r}$. If $F_{0}^{r+1}$ were a third such $(r+1)$-face, then aff $F_{0}^{r+1}=L^{r+1}$ because $h_{0}$ is simple. So $F_{0}^{r+1}$ lies in $\mathscr{E} \mid L^{r+1}$ and on one side or the other of $h_{0} \cap L^{r+1}$. Thus $F_{0}^{r+1}$ is one of the two $(r+1)$-faces already found.

It will be convenient to call these two $(r+1)$-faces the parents of the given $r$-face $F^{r}$. Note that they lie in precisely the same hyperplanes of $\mathscr{E} \backslash\left\{h_{0}\right\}$ as does $F^{r}$, and they lie on the same side of each of the remaining hyperplanes of $\mathscr{E} \backslash\left\{h_{0}\right\}$ as does $F^{r}$. Their union is an $(r+1)$-face of $\mathscr{E} \backslash\left\{h_{0}\right\}$.

We say that a hyperplane $h$ is close to a simple hyperplane $h_{0}$ of an arrangement $\mathscr{E}$ when (a) $h$ is different from and parallel to $h_{0}$, (b) $h$ is simple in $\mathscr{E} \cup\{h\}$, and (c) the open slab $\left(h_{0}, h\right)$ between $h_{0}$ and $h$ contains no points of intersection of $\mathscr{E}$.

LemMA 6 (Slicing LeMma). Let $F^{r}$, where $0<r \leqslant d$, be an $r$-face of an arrangement $\mathscr{E}$, and suppose that $h_{0}$ is a simple hyperplane of $\mathscr{E}$ that meets but does not contain $F^{r}$ ( so that $h_{0} \cap$ relint $\left.F^{r}=\varnothing\right)$. Let $h$ be a hyperplane that is close to $h_{0}$ and on the same side of $h_{0}$ as relint $F^{r}$, and let $h^{+}, h^{-}$be the two (closed) halfspaces with edge $h$. Then $h$ meets relint $F^{r}$, and $F^{r} \cap h^{+}$and $F^{r} \cap h^{-}$are both $r$-faces of the arrangement $\mathscr{E} \cup\{h\}$.

Proof. If $F^{r}$ is unbounded in the direction normal to $h_{0}$, then $h$ surely meets relint $F^{r}$. Otherwise, let $t$ be the distance between $h_{0}$ and $h$ and $S$ the set of points of $F^{r}$ at maximum distance $t_{0}$ from $h_{0}$. By way of contradiction, suppose that $t_{0} \leqslant t$. The set $S$ is an $s$-face of $F^{r}$, and $0<s<r$ because the half-closed slab $\left(h_{0}, h\right]=$ $\left(h_{0}, h\right) \cup h$ contains no points of intersection of $\mathscr{E}$. According to Lemma 4 , the induced arrangement $\mathscr{E} \mid$ aff $S$ is nondegenerate, so there are hyperplanes $h_{i}, 1 \leqslant i \leqslant s$, 
in $\mathscr{E}$, so that $\cap\left\{h_{i} \cap\right.$ aff $\left.S: 1 \leqslant i \leqslant s\right\}$ is a point. But aff $S$ is the intersection of $d-s$ hyperplanes $h_{i}, s+1 \leqslant i \leqslant d$, in $\mathscr{E}$. Consequently $\cap\left\{h_{i}: 1 \leqslant i \leqslant d\right\}$ is a point of $\mathscr{E}$ that lies in the slab $\left(h_{0}, h\right]$, a contradiction. So $t_{0}>t$, and again $h$ surely meets relint $F^{r}$.

The slicing lemma says that when a new hyperplane $h_{1}$ is inserted close to a simple hyperplane $h_{0}$ in an arrangement $\mathscr{E}$, the new faces one expects to find are actually formed. The following lemma makes precise the intuition that $\mathscr{E} \mid h_{1}$ and $\mathscr{E} \mid h_{0}$ must be nearly the same when $h_{1}$ is close to $h_{0}$.

LEMMA 7. If a hyperplane $h_{1}$ is close to a simple hyperplane $h_{0}$ in an arrangement $\mathscr{E}$, there exists an incidence-preserving bijection $\theta$ of the faces of $\mathscr{E} \mid h_{0}$ to the faces of $\mathscr{E} \mid h_{1}$ that is canonical in the following sense: if $F^{r}$ is an r-face of $\mathscr{E} \mid h_{0}$, then $\theta\left(F^{r}\right)$ is an $r$-face of $\mathscr{E} \mid h_{1}$, and for each hyperplane $h$ of $\mathscr{E} \backslash\left\{h_{0}, h_{1}\right\}$ either $F^{r}$ and $\theta\left(F^{r}\right)$ lie on the same side of $h$ or they both lie in $h$. Moreover, $\theta\left(F^{r}\right)$ is bounded precisely when $F^{r}$ is bounded.

Proof. If $F^{r}$ is a given $r$-face in $\mathscr{E} \mid h_{0}$, let $F^{r+1}$ be the parent $(r+1)$-face that lies on the same side of $h_{0}$ as $h_{1}$. Then $h_{1}$ meets relint $F^{r+1}$, and we take $\theta\left(F^{r}\right)$ to be the $r$-face $h_{1} \cap F^{r+1}$. It is easy to verify that $\theta$ has the properties announced except possibly the last. In particular, it follows that if $h$ is close to $h_{0}$, then the arrangements $\mathscr{E} \mid h$ and $\mathscr{E} \mid h_{0}$ are combinatorially equivalent (see Grünbaum [5, p. 394], or Zaslavsky [10, p. 2]). Since if $h_{0}$ and $h_{1}$ have equations $\langle x, u\rangle=\alpha_{0}$ and $\langle x, u\rangle=\alpha_{1}$, respectively, the hyperplane $h_{t}$ with equation $\langle x, u\rangle=t \alpha_{0}+(1-t) \alpha_{1}$ is close to $h_{0}$ for each $t$ in $(0,1]$, it follows from what has already been proved that $\mathscr{E} \mid h$, is combinatorially equivalent to $\mathscr{E} \mid h_{0}$ for each $t$ in $[0,1]$. Consequently the arrangements $\mathscr{E} \mid h_{0}$ and $\mathscr{E} \mid h_{1}$ are isotopic, i.e., one can be deformed continuously into the other within their combinatorial equivalence class (the interpolating arrangements $\mathscr{E} \mid h$, give the isotopy). It follows immediately that $\theta\left(F^{r}\right)$ is bounded precisely when $F^{r}$ is.

We call $\theta$ the canonical isomorphism of $\mathscr{E} \mid h_{0}$ to $\mathscr{E} \mid h_{1}$.

5. The anatomy of a plaid. A plaid $\mathscr{P}^{d}(\mathbf{n})$ can be generated by adding $n_{i}-1$ hyperplanes, which we call $i$-laminae, parallel to each hyperplane $h_{i}$ in a generic frame $\mathscr{F}=\left\{h_{i}: 1 \leqslant i \leqslant s\right\}$, taking care to keep the arrangement simple. In this section we study the anatomy of a plaid to prepare for the geometric derivation of the increment formulas in $\S 6$.

It follows from the sweep argument (cf. Alexanderson and Wetzel $[\mathbf{1}, \mathbf{2}]$ ) or from Theorems A and C of Zaslavsky [10] that the $i$-laminae can be translated as desired without changing the face counts as long as the final arrangement is again simple. In particular, we may, and do, assume that the $i$-laminae all lie on the same side of the frame hyperplane $h_{i}$. Write $h_{i 1}=h_{i}$, and name the $i$-laminae $h_{i j}, 2 \leqslant j \leqslant n_{i}$, in order from $h_{i}$. By further translations, if needed, we arrange for $h_{i j}$ to be close to $h_{i, j-1}$ (in the sense of $\S 4$ ) in $\mathscr{P}^{d}(\mathbf{n})$ for each $i$ and $j$ with $1 \leqslant i \leqslant s$ and $2 \leqslant j \leqslant n_{i}$. When these conditions are satisfied, we call the plaid narrow. For the remainder of this section, we assume that $\mathscr{P}=\mathscr{P}^{d}(\mathbf{n})$ is a given narrow plaid with Steiner data $\mathbf{n}$. 
To facilitate the geometric description, we introduce the "signature" of an $r$-face. For each frame hyperplane $h_{i}$, let $h_{i}^{+}$be the halfspace with face $h_{i}$ that contains the $i$-laminae, and let $h_{i}^{-}$be the opposite halfspace; if $n_{i}=1$, the selection of $h_{i}^{+}$can be made arbitrarily. For $1<j \leqslant n_{i}$, label the halfspaces $h_{i j}^{+}$and $h_{i j}^{-}$in such a way that $h_{i} \subseteq h_{i j}^{-}$. Note that then $h_{i k}^{+} \subseteq h_{i j}^{+}$when $1 \leqslant j \leqslant k \leqslant n_{i}$.

The signature $\varepsilon=\varepsilon\left(F^{r}\right)$ of an $r$-face of $\mathscr{P}$ is the $s$-vector $\left\langle\mathbf{b}_{i}: 1 \leqslant i \leqslant s\right\rangle$ of $n_{i}$-vectors $\mathbf{b}_{i}=\left\langle\varepsilon_{i j}: 1 \leqslant j \leqslant n_{i}\right\rangle$, where

$$
\varepsilon_{i j}=\left\{\begin{aligned}
1 & \text { if relint } F^{r} \subseteq h_{i j}^{+}, \\
0 & \text { if relint } F^{r} \subseteq h_{i j}, \\
-1 & \text { if relint } F^{r} \subseteq h_{i j}^{-}
\end{aligned}\right.
$$

We call the $n_{i}$-vector $\mathbf{b}_{i}$ a block of $\boldsymbol{\varepsilon}$, a zero block when $\varepsilon_{i j}=0$ for some $j$, a sign-change block when there is an index $j$ with $1 \leqslant j \leqslant n_{i}-1$ so that $\varepsilon_{i j}=1$ and $\varepsilon_{i, j+1}=-1$, and a constant block when $\varepsilon_{i j}=\varepsilon_{i 1}$ for each $j$ with $1 \leqslant j \leqslant n_{i}$. Finally, we write $z(\varepsilon)$ for the total number of zero entries in $\varepsilon$.

LeMma 8. Let $\varepsilon$ be the signature of an $r$-face $F^{r}$ in $\mathscr{P}$. Then

(a) $z(\varepsilon)=d-r$.

(b) The entries in each block $\mathbf{b}_{i}$ of $\boldsymbol{\varepsilon}$ are decreasing: $\varepsilon_{i j} \geqslant \varepsilon_{i k}$ when $1 \leqslant j \leqslant k \leqslant n_{i}$.

(c) Each block of $\varepsilon$ has at most one zero.

(d)

$$
F^{r}=\bigcap_{\varepsilon_{i j}=0} h_{i j} \cap \bigcap_{\varepsilon_{i j}=1} h_{i j}^{+} \cap \bigcap_{\varepsilon_{i j}=-1} h_{i j}^{-} .
$$

Proof. The assertions are all immediate consequences of the definition of a plaid and the indexing scheme.

Although every $r$-face of $\mathscr{P}$ has a signature, not every $s$-vector of blocks of 0 's, 1 's, and -1 's is the signature of an $r$-face, even if conditions (a), (b), and (c) of Lemma 8 are met, because the intersection in (d) might be void. The following two lemmas guarantee the realizability of certain modifications of realizable signatures.

LeMma 9. Suppose $\mathscr{P}$ contains an $r$-face $F^{r}$ with signature $\varepsilon$, and $1 \leqslant k \leqslant s$. If the s-vector $\overline{\boldsymbol{\varepsilon}}$ is obtained from $\boldsymbol{\varepsilon}$ by substituting for the kth block $\mathbf{b}_{k}$ of $\boldsymbol{\varepsilon}$ the block $\overline{\mathbf{b}}_{k}=\left\langle\bar{\varepsilon}_{k j}: 1 \leqslant j \leqslant n_{k}\right\rangle$ described in each instance below, then $\mathscr{P}$ contains an $r$-face $\bar{F}^{r}$ having signature $\bar{\varepsilon}$, and $F^{r}$ and $\bar{F}^{r}$ are both bounded or both unbounded.

(a) (Right zero-shift) If $\varepsilon_{k m}=0$ for some $m$ with $1 \leqslant m \leqslant n_{k}-1$, let

$$
\bar{\varepsilon}_{k j}=\left\{\begin{aligned}
1 & \text { for } 1 \leqslant j \leqslant m, \\
0 & \text { for } j=m+1, \\
-1 & \text { for } m+1<j \leqslant n_{k} .
\end{aligned}\right.
$$

(b) (Left zero-shift) If $\varepsilon_{k m}=0$ for some $m$ with $2 \leqslant m \leqslant n_{k}$, let

$$
\bar{\varepsilon}_{k j}=\left\{\begin{aligned}
1 & \text { for } 1 \leqslant j \leqslant m-2 \\
0 & \text { for } j=m-1, \\
-1 & \text { for } m-1<j \leqslant n_{k} .
\end{aligned}\right.
$$


(c) (Right sign-change shift) If $\varepsilon_{k m}=1$ and $\varepsilon_{k, m+1}=-1$ for some $m$ with $1 \leqslant m \leqslant n_{k}-2$, let

$$
\bar{\varepsilon}_{k j}=\left\{\begin{aligned}
1 & \text { for } 1 \leqslant j \leqslant m+1 \\
-1 & \text { for } m+1<j \leqslant n_{k} .
\end{aligned}\right.
$$

(d) (Left sign-change shift) If $\varepsilon_{k m}=1$ and $\varepsilon_{k, m+1}=-1$ for some $m$ with $2 \leqslant m \leqslant n_{k}-1$, let

$$
\bar{\varepsilon}_{k j}=\left\{\begin{aligned}
1 & \text { for } 1 \leqslant j \leqslant m-1 \\
-1 & \text { for } m-1<j \leqslant n_{k} .
\end{aligned}\right.
$$

Proof. We argue (a) and (c); the proofs of (b) and (d) are similar. In (a), the given $r$-face $F^{r}$ lies in $\mathscr{P} \mid h_{k m}$, and since $h_{k, m+1}$ is close to $h_{k m}$ in $\mathscr{P}$, it follows from Lemma 7 that the $r$-face $\theta\left(F^{r}\right)$ in $\mathscr{P} \mid h_{k, m+1}$ (where $\theta$ is the canonical isomorphism from $\mathscr{P} \mid h_{k m}$ to $\mathscr{P} \mid h_{k, m+1}$ ) has signature $\bar{\varepsilon}$ and is bounded precisely when $F^{r}$ is bounded.

We turn to (c). In the arrangement $\mathscr{P} \backslash\left\{h_{k, m+1}\right\}$, let $F_{0}^{r}$ be the parent $r$-face of the $(r-1)$-face $F^{r} \cap h_{k m}$ that lies in $h_{k m}^{+}$. Then $F_{0}^{r} \cap h_{k, m+2} \neq \varnothing$, and relint $F_{0}^{r} \subseteq$ $\left(h_{k m}, h_{k, m+2}\right)$. It follows that $h_{k, m+1}$ meets relint $F_{0}^{r}$, and the $r$-face $\bar{F}^{r}=F_{0}^{r} \cap$ $h_{k, m+1}^{+}$in $\mathscr{P}$ has signature $\bar{\varepsilon}$. If $F^{r}$ is bounded, then $F^{r} \cap h_{k m}$ is bounded; and since any hyperplane $h$ in $\left(h_{k m}, h_{k, m+2}\right]$ is close to $h_{k m}$ in $\mathscr{P} \backslash\left\{h_{k, m+1}\right\}$, it follows from Lemma 7 that $h \cap F_{0}^{r}$ is bounded. Thus $F_{0}^{r}$ is itself bounded, and so $\bar{F}^{r} \subseteq F_{0}^{r}$ is also bounded. The same reasoning from $\bar{F}^{r}$ back to $F^{r}$ establishes the converse.

LEMMA 10. For $0 \leqslant r \leqslant d-1$ and $1 \leqslant k \leqslant s$, if $\mathscr{P}$ contains an $r$-face $F^{r}$ with signature $\boldsymbol{\varepsilon}$ having a block $\mathbf{b}_{k}$ with a leading zero, and if $\overline{\boldsymbol{\varepsilon}}$ is formed from $\boldsymbol{\varepsilon}$ by replacing $\mathbf{b}_{k}$ by the $n_{k}$-vector $\overline{\mathbf{b}}_{k}$ in which the leading zero is replaced by 1 , then $\mathscr{P}$ contains an $(r+1)$-face $F^{r+1}$ with signature $\bar{\varepsilon}$; and conversely. If $F^{r+1}$ is bounded, so is $F^{r}$, and if $F^{r}$ is bounded and $n_{k}>1$, then $F^{r+1}$ is also bounded.

Proof. The parent $(r+1)$-face $F^{r+1}$ of $F^{r}$ that lies in $h_{k 1}^{+}$has the same signature as $F^{r}$ except that $\mathbf{b}_{k}$ is replaced by $\overline{\mathbf{b}}_{k}$. Conversely, if $\overline{\mathbf{b}}_{k}=\langle 1,-1, \ldots,-1\rangle$, then $F^{r+1}$ meets $h_{k 1}$ in an $s$-face $F^{s}$ for some $s$, and $s=r$ because $h_{k 1}$ is simple. The $r$-face $F^{r}$ plainly has signature $\varepsilon$.

If $F^{r+1}$ is bounded, so is $F^{r}$ because it is a facet of $F^{+1}$. Conversely, if $F^{r}$ is bounded and $n_{k}>1$, it follows from Lemma 7 that $h \cap F^{r+1}$ is bounded for every hyperplane $h$ in $\left(h_{k 1}, h_{k 2}\right.$ ], and so $F^{r+1}$ is bounded.

Finally, we need to associate the $r$-faces that are formed by the frame $\mathscr{F}$ of the plaid with certain $r$-faces that are formed by the plaid. To simplify the notation we shorten $\left\langle\varepsilon_{i 1}\right\rangle$ to $\varepsilon_{i 1}$, and we use the subscript " $f$ " to refer to the frame.

LEMMA 11. If $\varepsilon_{f}=\left\langle\varepsilon_{i 1}: 1 \leqslant i \leqslant s\right\rangle$ is the (frame) signature of an $r$-face $F_{f}^{r}$ formed by the frame $\mathscr{F}$ of $\mathscr{P}$ and if blocks $\mathbf{b}_{i}$ of length $n_{i}$ are defined by

$$
\mathbf{b}_{i}= \begin{cases}\langle 1,1, \ldots, 1\rangle & \text { if } \varepsilon_{i 1}=1, \\ \langle 0,-1, \ldots,-1\rangle & \text { if } \varepsilon_{i 1}=0 \\ \langle-1,-1, \ldots,-1\rangle & \text { if } \varepsilon_{i 1}=-1\end{cases}
$$


then there is an $r$-face $F^{r} \subseteq F_{f}^{r}$ of $\mathscr{P}$ with signature $\varepsilon=\left\langle\mathbf{b}_{i}: 1 \leqslant i \leqslant s\right\rangle$. Conversely, if an $r$-face $F^{r}$ of $\mathscr{P}$ has signature $\varepsilon$ in which every zero-block has a leading zero and every other block is constant, then $\varepsilon_{f}=\left\langle\varepsilon_{i 1}: 1 \leqslant i \leqslant s\right\rangle$ is the (frame) signature of an r-face $F_{f}^{r}$ such that $F^{r} \subseteq F_{f}^{r}$. The r-faces $F^{r}$ and $F_{f}^{r}$ are both bounded or both unbounded.

Proof. Suppose $F_{f}^{r}$ is an $r$-face of $\mathscr{F}$ with (frame) signature $\varepsilon_{f}=\left\langle\varepsilon_{i 1}: 1 \leqslant i \leqslant s\right\rangle$. If $\varepsilon_{i 1}=0$ or $\varepsilon_{i 1}=-1$, completing $\varepsilon_{i 1}$ to the full block $\mathbf{b}_{i}$ given by (10) obviously has no effect on $F_{f}^{r}$, because then the $i$-laminae do not meet $F_{f}^{r}$. In other words, $F_{f}^{r}$ is an $r$-face of the plaid

$$
\mathscr{P}_{0}=\mathscr{F} \cup \bigcup_{\substack{i=1 \\ \varepsilon_{i 1} \neq 1}}^{s} \pi_{i}
$$

with signature $\overline{\boldsymbol{\varepsilon}}=\left\langle\overline{\mathbf{b}}_{i}: 1 \leqslant i \leqslant s\right\rangle$, where $\overline{\mathbf{b}}_{i}=\mathbf{b}_{i}$ as given by (10) if $\varepsilon_{i 1} \neq 1$ and $\overline{\mathbf{b}}_{i}=1$ if $\varepsilon_{i 1}=1$. Suppose now that $\varepsilon_{i 1}=1$. Since $h_{i, j+1}$ is close to $h_{i j}$ for each $j$ with $1 \leqslant j \leqslant n_{i}-1$, it follows iteratively from the slicing lemma that $F_{i}^{r}=h_{i, n_{i}}^{+} \cap F_{f}^{r}$ is an $r$-face of $\mathscr{P}_{0} \cup \pi_{i}$ with signature $\overline{\boldsymbol{\varepsilon}}_{i}$ equal to $\bar{\varepsilon}$ except that $\overline{\mathbf{b}}_{i}=1$ is replaced by $\mathbf{b}_{i}$ as given by (10). Inserting each family $\pi_{i}$ with $\varepsilon_{i 1}=1$ completes the construction of the $r$-face $F^{r}$ of $\mathscr{P}$, and it is obvious that $F^{r} \subseteq F_{f}^{r}$.

Conversely, suppose $F^{r}$ is an $r$-face of $\mathscr{P}$ with signature $\varepsilon$ in which every zero-block has a leading zero and every other block is constant. Then

$$
\begin{gathered}
\varnothing \neq F^{r}=\bigcap_{\varepsilon_{i j}=0} h_{i j} \cap \bigcap_{\varepsilon_{i j}=1} h_{i j}^{+} \cap \bigcap_{\varepsilon_{i j}=-1} h_{i j}^{-} \\
\subseteq \bigcap_{\substack{i=1 \\
\varepsilon_{i 1}=0}}^{s} h_{i 1} \cap \bigcap_{\substack{i=1 \\
\varepsilon_{i 1}=1}}^{s} h_{i 1}^{+} \cap \bigcap_{\substack{i=1 \\
\varepsilon_{i 1}=-1}}^{s} h_{i 1}^{-}=F_{f}^{r}
\end{gathered}
$$

is the $r$-face of $\mathscr{F}$ having the desired (frame) signature. It remains to show that if $F_{f}^{r}$ is unbounded, then $F^{r}$ must also be unbounded. Let $h_{i}$ be a hyperplane of $\mathscr{F}$ so that the facet $F_{f}^{r} \cap h_{i}$ of $F_{f}^{r}$ is unbounded. If $\varepsilon_{i 1} \neq 1$, then $F_{f}^{r} \cap h_{i}$ is also a facet of $F^{r}$, and so $F^{r}$ is unbounded. If $\varepsilon_{i 1}=1$, the slicing lemma implies that $F^{r} \cap h_{i, n_{i}}$ is an unbounded facet of $F^{r}$, which consequently must be unbounded.

6. The increment formula. In this section we apply the geometric results of the previous section to count the $r$-faces formed by a plaid $\mathscr{P}^{d}(\mathbf{n})$. The formula involves sums of products of certain counting functions of $s$ with certain symmetric functions of the variables $n_{1}, n_{2}, \ldots, n_{s}$. We begin by defining these symmetric functions.

Let $x$ and $y$ be fixed integers with $0 \leqslant x \leqslant y \leqslant s$, let $S=\{1,2, \ldots, s\}$, let $Q$ be a (variable) $y$-element subset of $S$, and let $P$ be a (variable) $x$-element subset of $Q$. The symmetric functions we need are these:

$$
\tau_{x}^{y}(\mathbf{n})=\sum_{\substack{Q \subseteq S \\|Q|=y}} \sum_{P \subseteq Q} \prod_{p \in P} n_{p} \prod_{q \in Q \backslash P}\left(n_{q}-1\right) .
$$

In particular, $\tau_{j}^{j}(\mathbf{n})=\sigma_{j}(\mathbf{n})$, and

$$
\tau_{0}^{y}(\mathbf{n})=\sum_{\substack{Q \subseteq S \\|Q|=y}} \prod_{q \in Q}\left(n_{q}-1\right) .
$$


THEOREM 12 (INCREMENT FORMULA). For each $r$ with $0 \leqslant r \leqslant d$, a plaid $\mathscr{P}^{d}(\mathbf{n})$ with Steiner data $\mathbf{n}$ partitions $\mathbb{E}^{d}$ into

$$
\begin{aligned}
\varphi_{r}\left(\mathscr{P}^{d}(\mathbf{n})\right)= & \sum_{k=0}^{d-r} G_{r}^{d-k}(s-k) \tau_{0}^{k}(\mathbf{n}) \\
& +\sum_{k=d-r+1}^{d} G_{d-k}^{d-k}(s-k) \tau_{d-r}^{k}(\mathbf{n})
\end{aligned}
$$

$r$-faces.

Proof. Without loss of generality we may suppose that the given plaid $\mathscr{P}$ is narrow. The $r$-faces formed by $\mathscr{P}$ fall naturally into two classes by signature. In class A we put those $r$-faces in whose signature every nonzero block is constant, and in class B we collect all the remaining $r$-faces. The $r$-faces in class A are generated by zero-shifting from the frame, while those in class B are produced by both zero-shifting and sign-change shifting. We count the two classes separately.

An $r$-face of class $\mathrm{A}$ is generated from the frame by zero-shifting in, say, $k$ blocks, so we suppose that $0 \leqslant k \leqslant d-r$. For each $k$-element subset $Q$ of $S=\{1,2, \ldots, s\}$, let $\mathscr{F}_{Q}=\left\{h_{q} \in \mathscr{F}: q \in Q\right\}$. Because the frame is generic, the $(d-k)$-flat $L^{d-k}=$ $\cap\left(\mathscr{F}_{Q}\right)$ is cut by the remaining $s-k$ frame hyperplanes in a generic $(d-k)$ arrangement $\left(\mathscr{F} \backslash \mathscr{F}_{Q}\right) \mid L^{d-k}$, which forms $G_{r}^{d-k}(s-k) r$-faces. (When $k=0, L^{d}=$ $\mathbb{E}^{d}$ and $\mathscr{F} \mid L^{d}=\mathscr{F}$; when $r=0$ and $k=d, L^{d-k}$ is a point and $G_{0}^{0}(s-d)$ $=1$.)

Each such $r$-face $F_{f}^{r}$ has (frame) signature of the form $\varepsilon_{f}=\left\langle\varepsilon_{i 1}: 1 \leqslant i \leqslant s\right\rangle$, where $d-r$ of the entries are zero, including $\varepsilon_{q 1}$ for each $q$ in $Q$. It is associated by Lemma 11 with an $r$-face $F^{r}$ of $\mathscr{P}$ with signature $\varepsilon=\left\langle\mathbf{b}_{i}: 1 \leqslant i \leqslant s\right\rangle$ whose blocks are given by (10), and in particular $\mathbf{b}_{q}$ has a leading zero for each $q$ in $Q$. Note that $F^{r}$ is of class A.

According to Lemma 9(a), we can identify new $r$-faces of $\mathscr{P}$ by zero-shifting to the right. Moving the zero in $\mathbf{b}_{q}$ locates $n_{q}-1$ new $r$-faces of $\mathscr{P}$, so altogether we have identified

$$
G_{r}^{d-k}(s-k) \prod_{q \in Q}\left(n_{q}-1\right)
$$

$r$-faces, all of class A, that are associated with $Q$. Summing over all the $k$-element subsets $Q$ of $S$ and then summing on $k$ counts

$$
\sum_{k=0}^{d-r} G_{r}^{d-k}(s-k) \sum_{\substack{Q \subseteq S \\|Q|=k}} \prod_{q \in Q}\left(n_{q}-1\right)=\sum_{k=0}^{d-r} G_{r}^{d-k}(s-k) \tau_{0}^{k}(\mathbf{n})
$$

$r$-faces of class A in $\mathscr{P}$. These $r$-faces are different because they plainly have different signatures.

Next we show that (14) counts all of the $r$-faces of class A. Let $F^{r}$ be such an $r$-face with signature $\varepsilon=\left\langle\mathbf{b}_{i}: 1 \leqslant i \leqslant s\right\rangle$, let $Q$ be the set of indices $i$ so that $\mathbf{b}_{i}$ is a zero-block but the zero in $\mathbf{b}_{i}$ is not a leading zero, and let $|Q|=k$. Plainly $0 \leqslant k \leqslant z(\varepsilon)=d-r$. Zero-shifting the zero to the left in each block $\mathbf{b}_{q}$ for $q$ in $Q$, 
we see from Lemma 9(b) that $\mathscr{P}$ has an $r$-face $\bar{F}^{r}$ with signature $\overline{\boldsymbol{\varepsilon}}=\left\langle\overline{\mathbf{b}}_{i}: 1 \leqslant i \leqslant s\right\rangle$, where

$$
\overline{\mathbf{b}}_{i}= \begin{cases}\mathbf{b}_{i} & \text { if } i \notin Q, \\ \langle 0,-1, \ldots,-1\rangle & \text { if } i \in Q .\end{cases}
$$

According to Lemma 11 there is an associated $r$-face $F_{f}^{r}$ formed by the frame that has (frame) signature $\varepsilon_{f}=\left\langle\bar{\varepsilon}_{i 1}: 1 \leqslant i \leqslant s\right\rangle$, where $\bar{\varepsilon}_{i 1}$ is the leading term of $\overline{\mathbf{b}}_{i}$. Clearly the given $r$-face $F^{r}$ arises from the frame $r$-face $F_{f}^{r}$ by the process described above, and so it is counted in (13) and hence in (14). Thus (14) enumerates all the $r$-faces of $\mathscr{P}$ of class A.

The $r$-faces of class B are a little more difficult to describe. Suppose that $d-r+1 \leqslant k \leqslant d$. For each $k$-element subset $Q$ of $S$ let $\mathscr{F}_{Q}=\left\{h_{q} \in \mathscr{F}: q \in Q\right\}$. Then, as before, the $(d-k)$-flat $L^{d-k}=\cap\left(\mathscr{F}_{Q}\right)$ is cut by the remaining $s-k$ frame hyperplanes in a generic $(d-k)$-arrangement $\left(\mathscr{F}_{\mathcal{F}}\right) \mid \mathscr{F}^{d-k}$, which forms $G_{d-k}^{d-k}(s-k)(d-k)$-faces.

Each such $(d-k)$-face $F_{f}^{d-k}$ has (frame) signature of the form $\varepsilon_{f}=\left\langle\varepsilon_{i 1}: 1 \leqslant i \leqslant\right.$ $s\rangle$, where $\varepsilon_{q 1}=0$ for each $q$ in $Q$. It is associated by Lemma 11 with a $(d-k)$-face $F^{d-k}$ of $\mathscr{P}$ with signature $\boldsymbol{\varepsilon}=\left\langle\mathbf{b}_{i}: 1 \leqslant i \leqslant s\right\rangle$ whose blocks are given by (10), and in particular $\mathbf{b}_{q}$ has a leading zero for each $q$ in $Q$.

Let $P$ be a (variable) $(d-r)$-element subset of $Q$. For each $q$ in $Q \backslash P$ we replace the block $\mathbf{b}_{q}=\langle 0,-1, \ldots,-1\rangle$ of $\boldsymbol{\varepsilon}$ by $\overline{\mathbf{b}}_{q}=\langle 1,-1, \ldots,-1\rangle$, as in Lemma 10 , to identify an $r$-face $F^{r}$ in $\mathscr{P}$ whose dimension is $r$ because $(d-k)+(k-d+r)=r$ and whose signature $\overline{\boldsymbol{\varepsilon}}$ has a leading zero in each block $\overline{\mathbf{b}}_{p}$ for $p$ in $P$ and a leading sign-change in each block $\overline{\mathbf{b}}_{q}$ for $q$ in $Q \backslash P$ (if $n_{q}=1$, then $\overline{\mathbf{b}}_{q}=\langle 1\rangle$ ). Since $k>d-r,|Q \backslash P| \geqslant 1$, and $F^{r}$ is an $r$-face of class B.

According to Lemma 9(a), (c) we can identify new $r$-faces of $\mathscr{P}$ by zero-shifting and sign-change shifting to the right. The zero in a block $\overline{\mathbf{b}}_{p}$ for $p$ in $P$ can occupy any one of $n_{p}$ places, and a sign-change in $\overline{\mathbf{b}}_{q}$ for $q$ in $Q \backslash P$ can occupy any one of $n_{q}-1$ places, so altogether we have identified

$$
G_{d-k}^{d-k}(s-k) \prod_{p \in P} n_{p} \prod_{q \in Q \backslash P}\left(n_{q}-1\right)
$$

$r$-faces, all of class B, that are associated with $P$ and $Q$. Summing first over the $(d-r)$-element subsets $P$ of $Q$, then over the $k$-element subsets $Q$ of $S$, and finally over $k$ counts

$$
\begin{gathered}
\sum_{k=d-r+1}^{d-r} G_{d-k}^{d-k}(s-k) \sum_{\substack{Q \subseteq S \\
|Q|=k|P|=d-r}} \sum_{\substack{P \subseteq Q \\
\mid P \in P}} \prod_{p} \prod_{q \in Q \backslash P}\left(n_{q}-1\right) \\
=\sum_{k=d-r+1}^{d-r} G_{d-k}^{d-k}(s-k) \tau_{d-r}^{k}(\mathbf{n})
\end{gathered}
$$

$r$-faces of class B in $\mathscr{P}$. They are all different because they have different signatures.

It remains only to show that (16) counts all of the $r$-faces of $\mathscr{P}$ of class B. Let $F^{r}$ be such an $r$-face with signature $\varepsilon=\left\langle\mathbf{b}_{i}: 1 \leqslant i \leqslant s\right\rangle$; let $P$ be the set of indices $i$ in $S$ so that $\mathbf{b}_{i}$ is a zero-block, then $|P|=d-r$; let $R$ be the set of indices $i$ in $S$ so that $\mathbf{b}_{i}$ 
is a sign-change block; and let $Q=P \cup R$, then $|Q|=k>d-r$. Shifting all the zeros and sign-changes in $\varepsilon$ to the left, we see from Lemma $9(\mathrm{~b})$, (d) that there is an $r$-face $\bar{F}^{r}$ in $\mathscr{P}$ with signature $\overline{\boldsymbol{\varepsilon}}=\left\langle\overline{\mathbf{b}}_{i}: 1 \leqslant i \leqslant s\right\rangle$, where

$$
\overline{\mathbf{b}}_{i}=\left\{\begin{array}{cl}
\langle 0,-1, \ldots,-1\rangle & \text { if } i \in P, \\
\langle 1,-1, \ldots,-1\rangle & \text { if } i \in R, \\
\mathbf{b}_{i} \text { if } i \in S \backslash Q &
\end{array}\right.
$$

According to Lemma 10 there is a $(d-k)$-face $F^{d-k}$ in $\mathscr{P}$ in whose signature every zero-block has a leading zero and every other block $\overline{\mathbf{b}}_{i}$ is $\mathbf{b}_{i}$ (and so is constant). That $(d-k)$-face $F^{d-k}$ corresponds to a $(d-k)$-face $F_{f}^{d-k}$ formed by the frame, by the process of Lemma 11.

Now plainly the original $r$-face $F^{r}$ is included among those class $\mathrm{B} r$-faces that arise from the frame $(d-k)$-face $F_{f}^{d-k}$ by the process described above, and so it is counted in (15) and hence in (16). Thus (16) enumerates all the $r$-faces of $\mathscr{P}$ of class B.

Every $r$-face of $\mathscr{P}$ being of class A or class B, the formula of the theorem follows.

In the case of a skew plaid $\mathscr{S}^{d}(m)$, the functions $\tau_{x}^{l}(\mathbf{n})$, given by $(11)$, specialize as follows:

$$
\tau_{x}^{y}(m, 1, \ldots, 1)=\left\{\begin{array}{l}
(m-1)\left(\begin{array}{l}
s-1 \\
x-1
\end{array}\right)+\left(\begin{array}{l}
s \\
x
\end{array}\right) \text { if } y=x, \\
(m-1)\left(\begin{array}{c}
s-1 \\
x
\end{array}\right) \\
0 \text { if } y \geq x+2
\end{array} \text { if } y=x+1,\right.
$$

Then the increment formula (12) for $\mathscr{S}^{d}(m)$ becomes

$$
\begin{aligned}
\varphi_{r}\left(\mathscr{S}^{d}(m)\right)= & G_{r}^{d}(s)+(m-1) G_{r}^{d-1}(s-1) \\
& +(m-1)\left(\begin{array}{c}
s-1 \\
d-r
\end{array}\right) G_{r-1}^{r-1}(s-d+r-1) .
\end{aligned}
$$

Equating (17) with (6) gives the following curious identity, which, however, is not difficult to prove directly:

$$
\begin{gathered}
G_{r}^{d-1}(s-1)+\left(\begin{array}{c}
s-1 \\
d-r
\end{array}\right) G_{r-1}^{r-1}(s-d+r-1) \\
\quad=\sum_{j=d-r}^{d} \eta^{d-j}\left(\begin{array}{c}
j \\
d-r
\end{array}\right)\left(\begin{array}{l}
s-1 \\
j-1
\end{array}\right) .
\end{gathered}
$$

7. Algebraic arguments. The derivation of the increment formula (12) presented above is entirely geometric. In this section we sketch an ex post facto algebraic reduction of the right side of (12) to the right side of (3).

We begin by expressing $\tau_{\dot{x}}^{y}(\mathbf{n})$ in terms of the elementary symmetric functions $\sigma_{j}(\mathbf{n})$.

LEMMA 13. For $0 \leqslant x \leqslant y \leqslant s$,

$$
\tau_{\dot{x}}^{y}(\mathbf{n})=\sum_{j=x}^{y}(-1)^{y-j}\left(\begin{array}{l}
j \\
x
\end{array}\right)\left(\begin{array}{l}
s-j \\
y-j
\end{array}\right) \sigma_{j}(\mathbf{n}) .
$$


Proof. Expanding the innermost product in the definition (11) and reordering the sums, we see successively that

$$
\begin{aligned}
& \tau_{x}^{y}(\mathbf{n})= \sum_{\substack{Q \subseteq S \\
|Q|=y}} \sum_{\substack{P \subseteq Q \\
|P|=x}} \prod_{p \in P} n_{p} \sum_{z=0}^{y-x} \sum_{\substack{R \subseteq Q \backslash P \\
|R|=z}}(-1)^{y-x-z} \prod_{r \in R} n_{r} \\
&= \sum_{z=0}^{y-x}(-1)^{y-x-z} \sum_{\substack{Q \subseteq S \\
|Q|=y}} \sum_{\substack{P \subseteq Q \\
|P|=x}} \sum_{\substack{R \subseteq Q \backslash P \\
|R|=z}} \prod_{t \in P \cup R} n_{t} \\
&= \sum_{z=0}^{y-x}(-1)^{y-x-z} \sum_{\substack{P \subseteq S \\
|P|=x}} \sum_{\substack{R \subseteq S \backslash P|=z\\
| R \mid=P}} \prod_{t \in P \cup R} n_{t} \sum_{P \cup R \subseteq Q \subseteq S} 1 \\
&= \sum_{z=0}(-1)^{y-x-z}\left(\begin{array}{c}
S-x-z \\
y-x-z
\end{array}\right) \\
& \sum_{\substack{T \subseteq S \\
|T|=x+y}} \prod_{t \in T} n_{t} \sum_{\substack{P \subseteq T \\
|P|=x}} 1 \\
&= \sum_{z=0}^{y-x}(-1)^{y-(x+z)}\left(\begin{array}{c}
S-(x+z) \\
y-(x+z)
\end{array}\right)\left(\begin{array}{c}
x+y \\
x
\end{array}\right) \sigma_{x+y}(\mathbf{n}),
\end{aligned}
$$

which is (18).

To give a geometric interpretation, we call a plaid a grid if $s=d$, so a grid is the simplest possible plaid in the sense that it has only as many parallel families as are needed to make the arrangement nondegenerate. The bounded figure formed by a grid is a (possibly degenerate) parallelotope that is dissected by hyperplanes parallel to its facets.

COROLlaRY 14. For each $r, 0 \leqslant r \leqslant d$, a grid in $\mathbb{E}^{d}$ with Steiner data $\mathbf{n}$ forms $\tau_{d-r}^{d}(\mathbf{n})$ bounded $r$-cells.

This follows immediately from (18) and (2).

In the algebraic reduction of (12) to (3) we shall need the following elementary combinatorial identity (Gould [4, formula 3.119]): For any nonnegative integers $a$ and $b$,

$$
\sum_{k=a}^{b}(-1)^{k}\left(\begin{array}{l}
k \\
a
\end{array}\right)\left(\begin{array}{l}
b \\
k
\end{array}\right)=(-1)^{a} \delta_{a b}
$$

We turn now to that reduction. The right side $R$ of (12),

$$
R=\sum_{k=0}^{d-r} G_{r}^{d-k}(s-k) \tau_{0}^{k}(\mathbf{n})+\sum_{k=d-r+1}^{d} G_{d-k}^{d-k}(s-k) \tau_{d-r}^{k}(\mathbf{n}),
$$

in which (from (4)),

$$
G_{r}^{d}(n)=\left(\begin{array}{c}
n \\
d-r
\end{array}\right) \sum_{t=0}^{r} \eta^{r-t}\left(\begin{array}{c}
n-d+r \\
t
\end{array}\right),
$$

can be written, using (18), in the form $R=\sum_{j=0}^{d} \alpha_{j} \sigma_{i}(\mathbf{n})$, where for $0 \leqslant j \leqslant d-r-1$,

$$
\alpha_{j}=\sum_{t=0}^{r} \eta^{r-t}\left(\begin{array}{c}
s-d+r \\
t
\end{array}\right) \sum_{k=j}^{d-r}(-1)^{k-j}\left(\begin{array}{c}
s-k \\
d-r-k
\end{array}\right)\left(\begin{array}{c}
s-j \\
s-k
\end{array}\right)
$$


and for $d-r \leqslant j \leqslant d$,

$$
\alpha_{j}=\left(\begin{array}{c}
j \\
d-r
\end{array}\right) \sum_{k=j}^{d}(-1)^{k-j}\left(\begin{array}{c}
s-j \\
k-j
\end{array}\right) \sum_{i=k}^{d} \eta^{d-i}\left(\begin{array}{c}
s-k \\
i-k
\end{array}\right) .
$$

The inner sum in (20) is equal to

$$
(-1)^{s-j} \sum_{x=s-d+r}^{s-j}(-1)^{x}\left(\begin{array}{c}
x \\
s-d+r
\end{array}\right)\left(\begin{array}{c}
s-j \\
x
\end{array}\right)=0
$$

according to (19), so that $\alpha_{j}=0$ for $0 \leqslant j \leqslant d-r$. Reversing the order of summation in (21) leads to

$$
\alpha_{j}=\left(\begin{array}{c}
j \\
d-r
\end{array}\right) \sum_{i=j}^{d} \eta^{d-i} \sum_{k=j}^{i}(-1)^{k-j}\left(\begin{array}{c}
s-k \\
s-i
\end{array}\right)\left(\begin{array}{c}
s-j \\
s-k
\end{array}\right) .
$$

It follows from (19) that the inner sum is different from zero only when $i=j$, and then its value is one. So for $d-r \leqslant j \leqslant d, \alpha_{j}=\eta^{d-j}\left(d_{d-r}^{j}\right)$. Consequently, $R$ is equal to the right side of (3), as claimed.

8. Zaslavsky's formula. In June 1978, prompted by a preprint of an early version of this paper, Zaslavsky sent the second author an ingenious generating function argument that yields a face-count formula for a plaid superficially similar to the increment formula (12). We include Zaslavsky's formula here with his kind permission.

In stating the formula we continue the convention set earlier that $G_{r}^{d}(n)=0$ when $0 \leqslant r \leqslant d$ is false. Zaslavsky showed that

$$
\varphi_{r}\left(\mathscr{P}^{d}(\mathbf{n})\right)=\sum_{k=0}^{d}\left\{\sum_{j=r}^{k}\left(\begin{array}{l}
k \\
j
\end{array}\right) G_{r-j}^{d-k}(s-k)\right\} \tau_{0}^{k}(\mathbf{n}) .
$$

This formula seems not to be an easy consequence of the increment formula (12) because the underlying geometric considerations are quite different, but it is not difficult to deduce it algebraically from (3).

We begin by expressing (3) in terms of the $\tau_{0}^{k}(\mathbf{n})$. According to (18), for each $k$ with $0 \leqslant k \leqslant s$,

$$
\tau_{0}^{k}(\mathbf{n})=\sum_{j=0}^{k}(-1)^{k-j}\left(\begin{array}{c}
s-j \\
s-k
\end{array}\right) \sigma_{j}(\mathbf{n}) .
$$

It follows from a well-known inverse pair (Riordan [7, formula 4a on p. 45]) that for each $j$ with $0 \leqslant j \leqslant s$,

$$
\boldsymbol{\sigma}_{j}(\mathbf{n})=\sum_{k=0}^{j}\left(\begin{array}{c}
s-k \\
s-j
\end{array}\right) \tau_{0}^{k}(\mathbf{n}) .
$$

Consequently, (3) can be written in the form

$$
\varphi_{r}\left(\mathscr{P}^{d}(\mathbf{n})\right)=\sum_{k=0}^{d}\left\{\sum_{j=d-r}^{d} \eta^{d-j}\left(\begin{array}{c}
j \\
d-r
\end{array}\right)\left(\begin{array}{c}
s-k \\
s-j
\end{array}\right)\right\} \tau_{0}^{k}(\mathbf{n}),
$$

a formula that is of some interest in its own right. 
To prove Zaslavsky's formula (22) we reduce it to (23). Substituting (3) for $G_{r-j}^{d-k}(s-k)$ into the coefficient of $\tau_{0}^{k}(\mathbf{n})$ in (22) and reversing the order of summation leads to

$$
\begin{gathered}
\sum_{m=d-r}^{d} \eta^{d-m}\left(\begin{array}{c}
s-k \\
m-k
\end{array}\right)^{m-d+r}\left(\begin{array}{l}
k \\
j=0
\end{array}\right)\left(\begin{array}{c}
m-k \\
(m-d+r)-j
\end{array}\right) \\
=\sum_{m=d-r}^{d} \eta^{d-m}\left(\begin{array}{c}
s-k \\
m-k
\end{array}\right)\left(\begin{array}{c}
m \\
d-r
\end{array}\right)
\end{gathered}
$$

by the Vandermonde convolution formula (Gould [4, formula 3.1]). Thus (22) is transformed into (23).

\section{REFERENCES}

1. G. L. Alexanderson and John E. Wetzel, Arrangements of planes in space, Discrete Math. 34 (1981), 219-240; Erratum, Discrete Math. 45 (1983), 140.

2. Simple partitions of space, Math. Mag. 51 (1978), 220-225.

3. R. C. Buck, Partition of space, Amer. Math. Monthly 50 (1943), 541-544.

4. H. W. Gould, Combinatorial identities, rev. ed., publ. by the author, 1972.

5. Branko Grünbaum, Convex polytopes, Interscience, New York, 1967.

6. P. McMullen and G. C. Shephard, Convex polytopes and the upper bound conjecture, London Math. Soc. Lecture Note Series 3, Cambridge Univ. Press, Cambridge, 1971.

7. John Riordan, Combinatorial identities, Krieger, Huntington, N. Y., 1979.

8. J. Steiner, Einige Gesetze über die Theilung der Ebene und des Raumes, J. Reine Angew. Math. 1 (1826), 349-364.

9. Thomas Zaslavsky, A combinatorial analysis of topological dissections, Adv. in Math. 25 (1977), 267-285.

10. Facing up to arrangements: Face-count formulas for partitions of space by hyperplanes, Mem. Amer. Math. Soc. No. 154 (1975).

Department of Mathematics, University of Santa Clara, Santa Clara, California 95053

Department of Mathematics, University of Illinois at Urbana-Champaign, 1409 WeSt Green STREeT, URBANA, IllinoIS 61801 